\title{
Spatial and Temporal Variation of Wideband Indoor Channels
}

\author{
David L. Ndzi, Nick Savage, and Boris Gremont \\ Department of Electronic and Computer Engineering, University of Portsmouth, Portsmouth PO1 3DJ, UK \\ Correspondence should be addressed to David L. Ndzi, david.ndzi@port.ac.uk
}

Received 25 March 2010; Accepted 9 July 2010

Academic Editor: Ananda Sanagavarapu Mohan

Copyright (C) 2010 David L. Ndzi et al. This is an open access article distributed under the Creative Commons Attribution License, which permits unrestricted use, distribution, and reproduction in any medium, provided the original work is properly cited.

\begin{abstract}
Extensive studies of the impact of temporal variations induced by people on the characteristics of indoor wideband channels are reported. Singular Value Decomposition Prony algorithm has been used to compute the impulse response from measured channel transfer functions. The high multipath resolution of the algorithm has allowed a detailed assessment of the shapes of individual multipath clusters and their variation in time and space in indoor channels. Large- and small-scale analyses show that there is a significant dependency of the channel response on room size. The presence of people in the channel has been found to induce both signal enhancements and fading with short-term dynamic variations of up to $30 \mathrm{~dB}$, depending on the number of people and their positions within the room. A joint amplitude and time of arrival model has been used to successfully model measured impulse response clusters.
\end{abstract}

\section{Introduction}

The rapid growth in the wireless communication market has led to the need to study, understand, and model all factors that can contribute to communication impairments. More than ever before, there is a greater need to understand fast variation in systems that are required to support an ever increasing high data rate transmission. Wireless Local Area Network (WLAN) system implementation has adopted MIMO techniques to achieve higher spectral efficiencies, throughput, and quality of service. The proposed overlay of WLAN hotspots on cellular networks for high data rate mobile services has galvanised interest within the research community [1].

The limited bandwidth in the WLAN frequency bands mandates a thorough understanding and exploitation of all favourable propagation conditions for communication. This requires the development and refinement of channel models that could lead to the identification of channel characteristics that can further be exploited to achieve better spectral efficiency through new system design techniques. The channel model must incorporate both spatial and temporal channel variations [2] so as to provide a comprehensive basis for performance evaluation and comparison of time diversity wireless communication systems, taking into account other channel parameters.
Despite the availability of many spatial models in open literature, few incorporate the short-term dynamic channel fading. Research such as that performed by Chen and Dubey [3] have incorporated temporary aspects into their models, but this is usually limited to transceiver movement rather than the movement of people in the channel. More recently Gupta et al. [4] have investigated the effect of people movement on the channel response. They found that the presence of people in the propagation channel can increase channel capacity as well as reduce it. Their research was very methodical and was not representative of a typical working environment. Understanding the correlation between the spatial, angular, and temporal variations could improve the evaluation of space-angle and space-time systems [5]. Many reported models on impulse response have adopted the statistical model proposed by Saleh and Valenzuala [6]. The Saleh-Valenzuela model assumes that multipath components arrive in clusters and that the intercluster and intracluster arrival time can be modelled as a Poisson process, and the amplitudes have an exponential distribution. Different extensions of this model have been reported but the limited time-delay resolution of most measurement systems used has meant that the cluster shapes and models have not been examined in greater detail. 
The objective of this paper is fourfold. Firstly, the paper describes a comprehensive measurement and analysis of indoor wideband channel at three distinct frequencies. Secondly, large- and small-scale results and analysis of signal variations within different indoor environments are presented to provide a more comprehensive representation of path loss within the different channels and their relation to the room and its volume. Thirdly, a detailed analysis of the dynamic temporal channel variation induced by the presence of people within the channel is discussed. Fourthly, detailed analysis of the impulse response within the indoor channel is presented. Comparisons are made between the measured data and predictions using Saleh-Valenzuela models. The high time-delay resolution of the system used allows a more comprehensive assessment of the structure of the delay power profile and individual clusters. A joint amplitude and delay probability density function is proposed as a means to estimate the amplitude of impulses/clusters in any given channel.

This paper is organised as follows. Section 2 describes the measurement system, setup, environment, and the data processing procedure used. Section 3 presents the results of large-scale and small-scale spatial variations of signal power. Results of more than two weeks of continuous measurements to assess the impact of people on communication channels are described in Section 4. The evaluation and modelling of the delay power profile (DPP) and the individual clusters are presented in Section 5. Finally, in Section 6 relevant conclusions are drawn.

\section{Indoor Measurement Setup and Environment}

The experimental campaign was conducted using a wideband channel sounder, the details, and design principles of which are given in [7]. The channel sounder transmits a $31.25 \mathrm{MHz}$ bandwidth rectangular spectrum at carrier frequencies of $1.3 \mathrm{GHz}, 2 \mathrm{GHz}$, and $11.6 \mathrm{GHz}$. Although transmission is simultaneous, only one frequency is received at any one time. A $30 \mathrm{~Hz}$ channel sampling rate was used. The transmitted power and the beamwidth of the directional antennas used at the receivers are summarised in Table 1. The transmitting antennas were all omnidirectional. Unless otherwise specified, the transmitting and receiving antennas were mounted at heights of $2 \mathrm{~m}$ and $1.6 \mathrm{~m}$, respectively, above the mean floor level. During all measurements, the transmitter remained fixed in one position whilst the receiver was moved from one measurement point to another. At each position, a total of 512 channel responses were acquired. Measurements were also conducted with omni-directional receiving antennas at every position.

Figure 1 shows the plan of two floors in the building (Anglesea) where measurements were taken. The building structure is made of re-enforced concrete. The volumes of the rooms and corridors where measurements were carried out are given in Table 2. Corridors 1 and 2 have the same shape and dimensions, but one wall of corridor 2 is lined with metal lockers.
TABLE 1: Beamwidths of antennas used.

\begin{tabular}{lcc}
\hline $\begin{array}{l}\text { Frequency } \\
(\mathrm{GHz})\end{array}$ & TX Power $(\mathrm{dBm})$ & $\begin{array}{c}\text { Receiving Antenna } \\
\text { Beamwidth }\end{array}$ \\
\hline 1.3 & 17 & $18^{\circ}$ \\
2 & 22 & $70^{\circ}$ \\
11.6 & 16 & $20^{\circ}$ \\
\hline
\end{tabular}

Singular Value Decomposition Prony (SVDP) algorithm that improves multipath resolution by factors of up to 6 compared to the Fast Fourier Transform (FFT) has been used to compute the impulse response from measured channel transfer function [8]. Performance analysis showed that SVDP has a time delay resolution of up to $5 \mathrm{~ns}$ for a $31.25 \mathrm{MHz}$ bandwidth. The measured channel impulse response (CIR) can be represented by (1). This describes each $k$ component as having a time delay $\tau_{k}$, amplitude $\alpha_{k}$, and phase $\theta_{k}$. The time delay can be estimated if the length of the propagation path is known. However, it is often modelled as a Poisson process

$$
h(t)=\sum_{k=0}^{N} \alpha_{k} \delta\left(t-\tau_{k}\right) e^{j \theta_{k}} .
$$

Overall, the channel was considered to be time invariant during the measurement period $(1 \mathrm{~ms})$ of each channel transfer function. This assumption is considered to be valid even in the presence of people in the channel. Furthermore, results reported in [9] show that the coherence time of the channel is greater than $1 \mathrm{~ms}$.

\section{Path Loss and Small-Scale Variation}

A number of models have been proposed for path loss, which include the dual gradient model [10], the ITU-R model [11], and the modified path loss (MPL) model [12]. The two latter models are represented by (2). In these models, $f$ is the frequency (MHz), $\lambda$ is the wavelength (in centimetres), $d$ is the distance from the transmitter to the receiver (in metres), $L_{f}$ is the floor attenuation factor, $n_{f}$ is the number of floors, and $n$ is a fitted loss factor (in free space $n=2$ ) $[12,13]$. Examples of signal power variation with antenna separation are shown in Figure 2. The values of $n$ for the modified path loss model were found to vary between 1.7 and 3, with a mean of 2.4 and a standard deviation of 0.3 . The MPL model has been found to provide the best description of path loss for the different room sizes, shapes, and uses. The values of $n$ for the different locations are summarized in Table 2:

$$
\begin{gathered}
L_{T}=20 \log _{10} f+n \log _{10} d+n_{f} L_{f}-28, \\
L_{T}=10 n \log _{10}\left(\frac{4 \pi d}{\lambda}\right)+n_{f} L_{f} .
\end{gathered}
$$

The results show that signal propagation along corridors suffers less attenuation compared to propagation within rooms. Despite the two corridors, 1 and 2, having the same dimensions and layout, the differences in the values of $n$ (Table 2) emphasise the importance of taking into account 


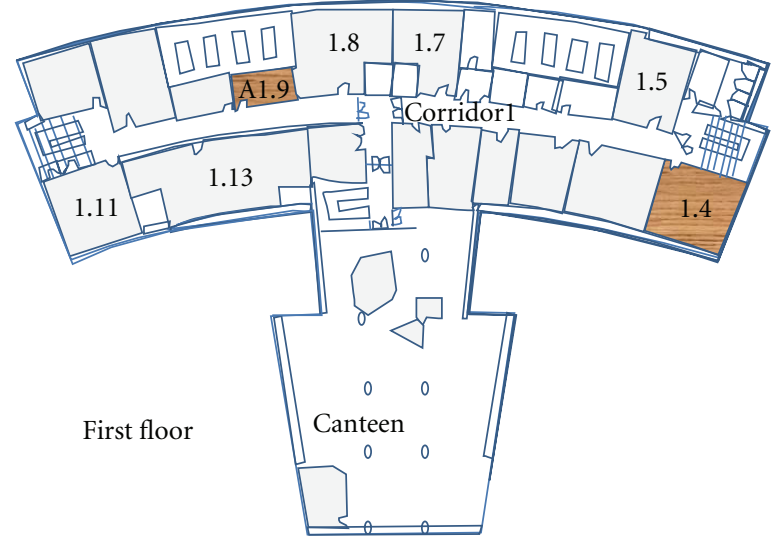

(a)

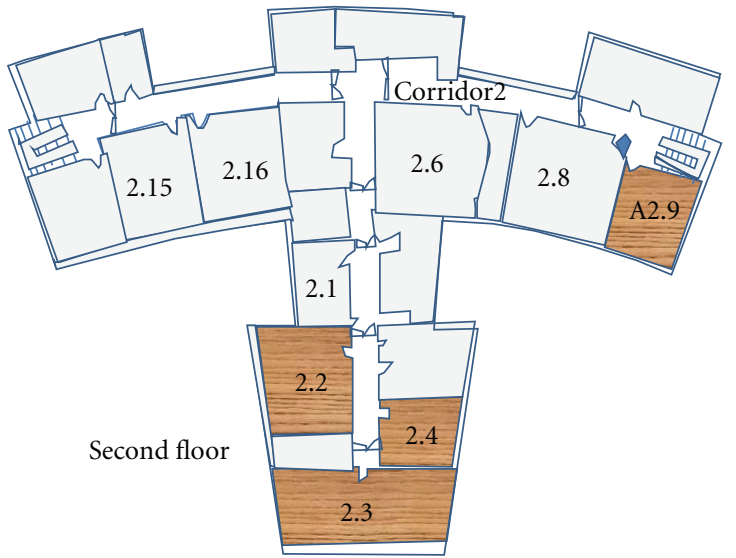

(b)

FIgURE 1: Plan of (a) first floor and (b) second floor of Anglesea Building showing measurement locations.

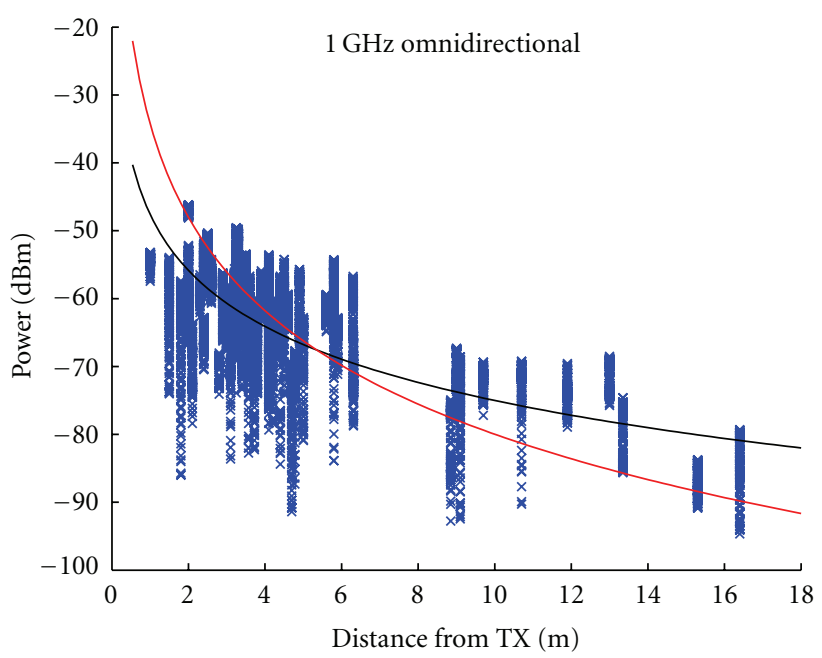

$\times \quad$ Measured data

- Fitted model (nLf), $n=2.74$, MSE $=6.7262$

— Fitted model (ITU-R), $n=45.7$, MSE $=9.0911$

(a)

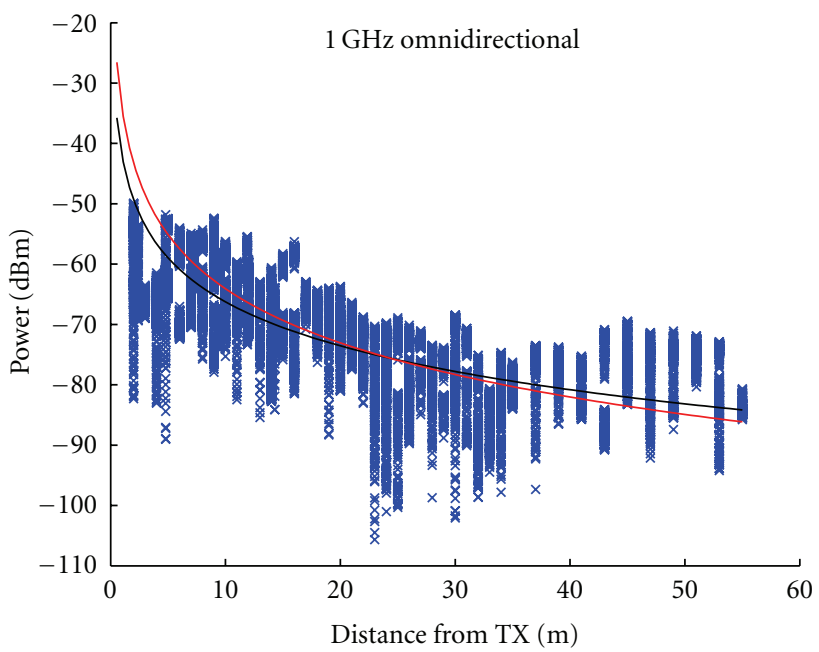

$\times \quad$ Measured data

- Fitted model (nLf), $n=2.42, \mathrm{MSE}=6.664$

- Fitted model (ITU-R), $n=29.8, \mathrm{MSE}=7.5999$

(b)

FIGURE 2: Signal power variation with distance at $1.3 \mathrm{GHz}$ for measurements (a) within rooms and (b) along a corridor.

TABLE 2: Values of path loss exponent $(n)$ for measurements in different indoor environments.

\begin{tabular}{|c|c|c|c|c|c|c|c|}
\hline Room & Volume & $\begin{array}{c}1.3 \mathrm{GHz} \\
\text { Omni-directional }\end{array}$ & $\begin{array}{c}1.3 \mathrm{GHz} \\
\text { Directional }\end{array}$ & $\begin{array}{c}2 \mathrm{GHz} \\
\text { Omni-directional }\end{array}$ & $\begin{array}{c}2 \mathrm{GHz} \\
\text { Directional }\end{array}$ & $\begin{array}{c}11.647 \mathrm{GHz} \\
\text { Omni-directional }\end{array}$ & $\begin{array}{l}11.647 \mathrm{GHz} \\
\text { Directional }\end{array}$ \\
\hline Canteen & 1054.48 & 2.79 & 2.57 & 2.62 & 2.22 & 2.69 & 2.47 \\
\hline A 2.2 & 347.76 & 2.58 & 2.27 & 2.28 & 1.83 & 2.82 & 2.41 \\
\hline A 2.9 & 250.92 & 2.64 & 2.35 & 2.49 & 2.11 & 2.49 & 2.24 \\
\hline Staff room & 238.82 & 2.9 & 2.67 & 2.7 & 2.15 & 3.01 & 2.68 \\
\hline A1.4 & 217.6 & 2.65 & 2.47 & 2.39 & 2.08 & 2.77 & 2.43 \\
\hline A2.4 & 199.37 & 3.02 & 2.76 & 2.66 & 2.3 & 3.12 & 2.72 \\
\hline A1.9 & 79.06 & 2.65 & 2.94 & 2.57 & 2.29 & 3 & 2.78 \\
\hline Corridor 1 & $>1000$ & 2.49 & 2.25 & 2.3 & 1.98 & 2.52 & 2.23 \\
\hline Corridor 2 & $>1000$ & 2.37 & 2.09 & 2.02 & 1.51 & 2.42 & 1.97 \\
\hline
\end{tabular}




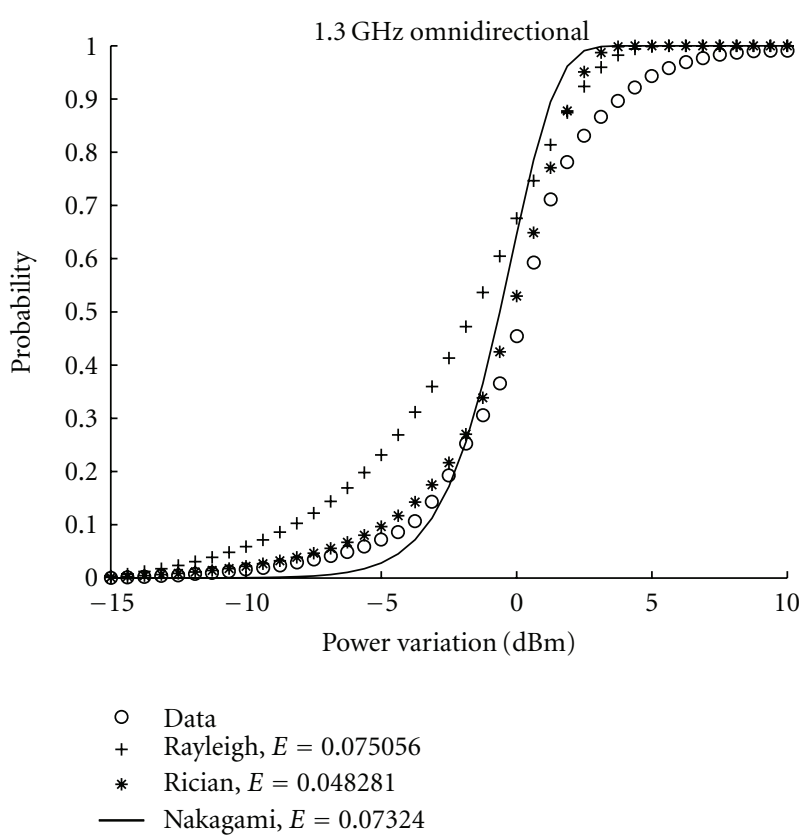

(a)

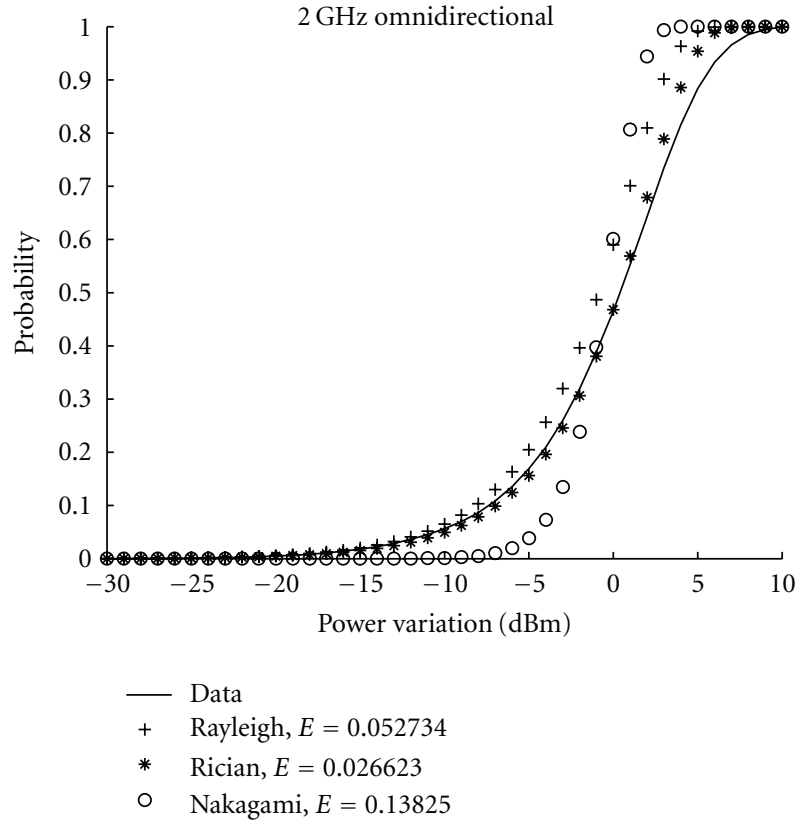

(b)

FIGURE 3: Small-scale signal variation in (a) canteen (large room) (b) A2.4 (small room).

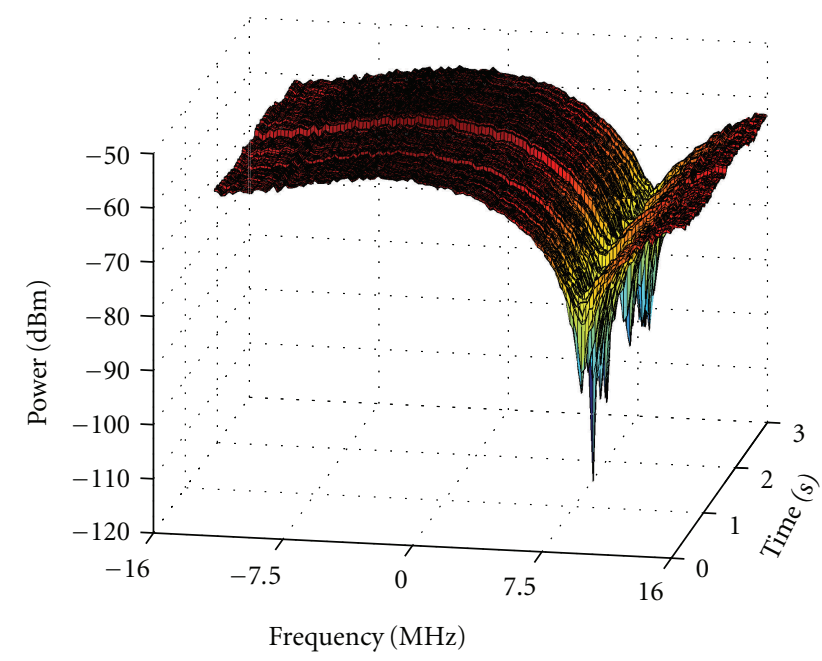

Figure 4: An example of frequency selective fading over time at $1.3 \mathrm{GHz}$ using directional antennas in a small room $(3.6 \times 3 \times 7.32)$.

the material on the walls in path loss calculations. Path loss in corridor 2 is smaller than free space loss at $2 \mathrm{GHz}$ and $11.6 \mathrm{GHz}$ when directional antennas are used at the receiver. At $1.3 \mathrm{GHz}$, the average path loss is marginally greater than free space loss. Narrow band path losses in corridors are significantly influenced by frequency selective fading. This is illustrated by the large variations at each measurement position in Figure 2(b).

WLAN network planning requires knowledge of signal attenuation by walls and floors, especially in a multifloor building to determine coverage and spectrum utilisation.
This knowledge is also required for the planning of outdoor to indoor (and vice versa) systems. In the building used, the walls are built with standard concrete material reenforced by supporting pillars. To determine the wall and floor attenuation values, measurements were conducted with the transmitter and receiver on the same side and then on opposite sides of the wall (floor). Measurements were also conducted to determine additional signal loss when the transmitter and receiver are placed in two different corridors that are perpendicular without LOS. The average attenuation at the 3 frequencies and antenna types are summarised in Table 3 . The attenuations were computed for both the modified path loss and the ITU-R models. The floor attenuation at $11.6 \mathrm{GHz}$ could not be measured accurately because the received signal levels were very close to the noise floor of the experimental system. The results show that higher attenuations are obtained with directional antenna. This can be attributed to the fact that directional antennas do not receive all the scatter power. Impulse response analysis showed that there were no dominant multipath components and the received signals were an aggregation of multiple scattered components.

Indoors to outdoors (and vice versa) measurements showed that the additional losses due to the walls were $22 \mathrm{~dB}, 33 \mathrm{~dB}$, and $50 \mathrm{~dB}$ at $1.3,2$, and $11.6 \mathrm{GHz}$, respectively. The results confirmed that total attenuation depends on the wall material, with rooms that have large windows showing smaller losses.

The small-scale variation was computed by considering every spectral line within the transmitted spectrum. This is equivalent to narrow band fading and provides evidence of signal variation across the band. In [14], small-scale fading 
TABLE 3: Fitted additional loss due to walls and floors (dB).

\begin{tabular}{|c|c|c|c|c|c|c|c|}
\hline & \multicolumn{2}{|c|}{$1.3 \mathrm{GHz}$} & \multicolumn{2}{|c|}{$2 \mathrm{GHz}$} & \multicolumn{2}{|c|}{$11.6 \mathrm{GHz}$} \\
\hline & & Omni-directional & Directional & Omni-directional & Directional & Omni-directional & Directional \\
\hline \multirow[t]{2}{*}{ Floor } & MPL & 33 & 40 & 40 & 58 & - & - \\
\hline & ITU-R & 36 & 42 & 42 & 55 & - & - \\
\hline \multirow[t]{2}{*}{ Wall } & MPL & 18 & 17 & 28 & 38 & 48 & 54 \\
\hline & ITU-R & 20 & 19 & 30 & 36 & 47 & 54 \\
\hline \multirow[t]{2}{*}{ Corner } & MPL & 20 & 13 & 27 & 25 & 47 & 50 \\
\hline & ITU-R & 24 & 15 & 29 & 22 & 48 & 51 \\
\hline
\end{tabular}
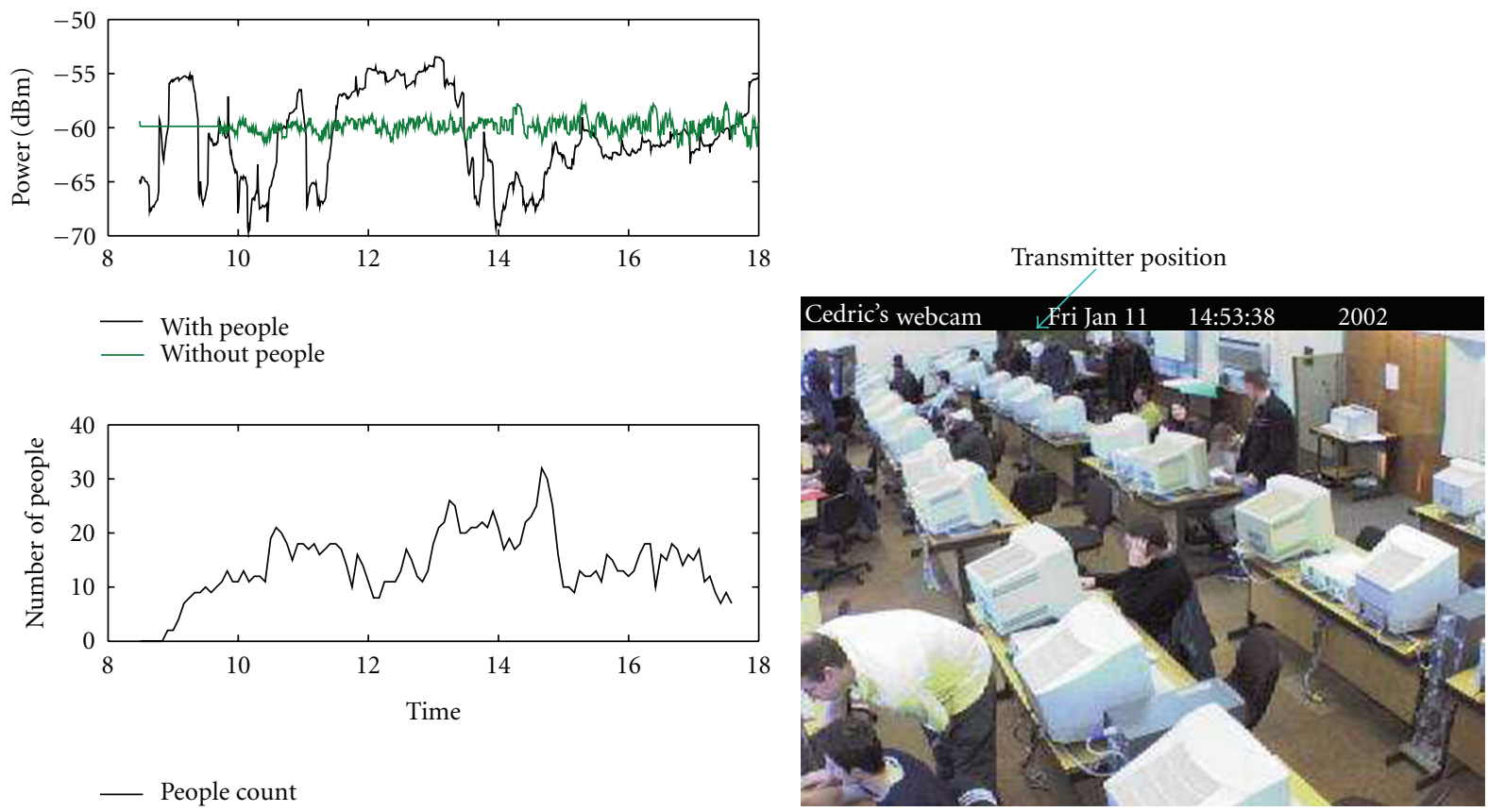

(a)

(b)

FIGURE 5: (a) Correlation between the number of people in the room and signal level at $2 \mathrm{GHz}$ and (b) an image of the indoor environment showing the view from the receiver position.

has been reported to be Rayleigh distributed but studies in [15] at $10 \mathrm{GHz}$ showed that Nakagami distribution provided the best fit to the measured data. Analysis of the measured data showed that at 1.3, 2 and $11.6 \mathrm{GHz}$ small-scale fading for LOS propagation is best described by Rice distribution as shown in Figure 3. The computed Rice K-factor decreases with increase in frequency.

The Nakagami probability density function is given by (3) where $x$ is the data and $\Omega$ is the scale parameter equal to the mean value of $x^{2}$, and $m$ is the Nakagami parameter. Rayleigh and Rice distributions are special cases of the Nakagami distribution when the Nakagami parameter $(m)$ is 1 and greater than 1 , respectively. The Rayleigh and Rice probability density functions are given by (4) and (5), respectively. This dependency on the value of $m$ means that the Nakagami distribution is likely to be seen as a much more general model for environments where there are large spatial changes in the amount of scattering from one position to another. This is more likely to be the case at frequencies greater than $10 \mathrm{GHz}$ than at lower frequencies,

$$
\begin{gathered}
p(x)=\frac{2 m^{m}}{\Gamma(m) \Omega^{m}} x^{2 m-1} e^{-(m / \Omega) x^{2}}, \\
p(x)=\frac{x}{\sigma^{2}} e^{\left(-x^{2} / 2 \sigma^{2}\right)} \quad \text { for } x \geq 0, \\
p(x)=\frac{x}{\sigma^{2}} e^{\left[-\left(x^{2}+a_{p k}^{2}\right) / 2 \sigma^{2}\right]} I_{0}\left(\frac{x a_{p k}}{\sigma^{2}}\right),
\end{gathered}
$$

where $I_{0}$ is the modified Bessel function of the first kind and zero order and $a_{p k}$ is the peak amplitude of the dominant signal component.

Figure 3 shows the fitting to the data measured in a large room (canteen with a volume of $1054.48 \mathrm{~m}^{3}$ ) and a small room (A2.4 with a volume of $199.37 \mathrm{~m}^{3}$ ). For large open rooms, the received signal power can be modelled using Rice distribution. In small size rooms multipath components travel over relatively short distances and can have amplitudes that are comparable to the LOS components. These result in severe frequency selective fading as shown in Figure 4. Fade depths of up to $40 \mathrm{~dB}$ have been measured in small rooms. 

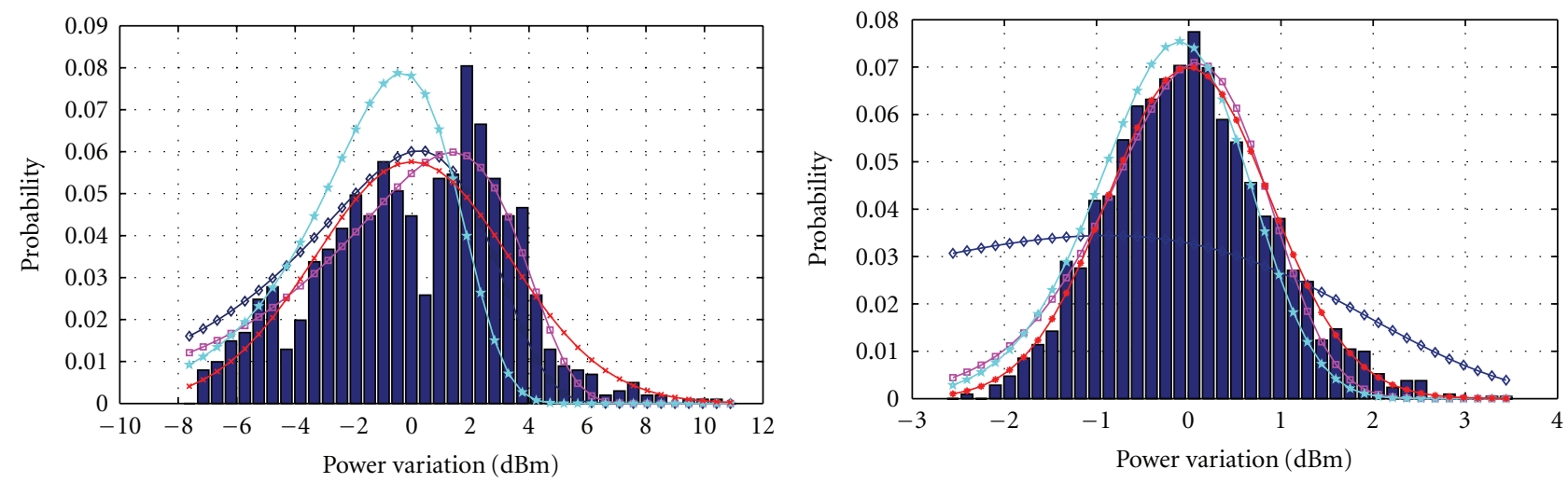

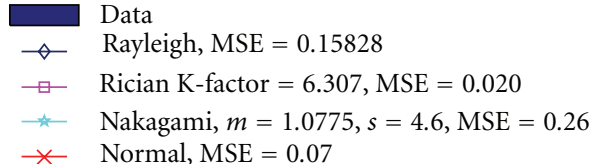

(a)

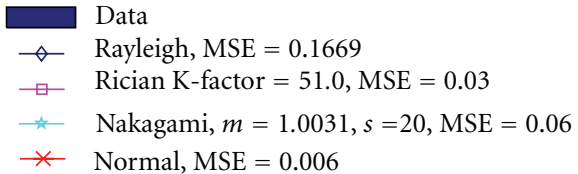

(b)

FIgURE 6: Probability density function of small-scale variation at $1.3 \mathrm{GHz}$ (a) with people and (b) without people, in the room.

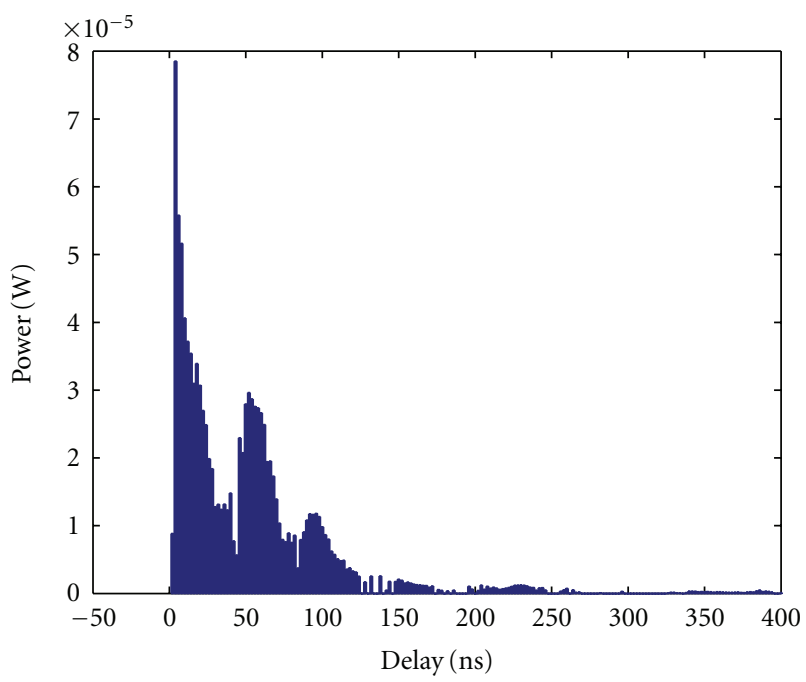

Figure 7: An example of a measured delay power profile, showing distinct clusters.

Greater frequency selective fading has also been obtained with directional antennas.

\section{Effect of People on Channel Response}

Extensive measurements were conducted in a large computer laboratory for time periods lasting more than 24 hours with both omni- and directional antennas at the receiver. The room was normally in use by students between 8:30 am and 9:00 pm. A video camera was mounted above the receiving antenna and the video images were used to correlate the measured signal levels with the number and positions of people in the room. Figure 5(a) shows an example of the correlation between the total number of people in the room and the measured signal levels in the presence and absence of people. Figure 5(b) shows an image of the room as seen from the receiver. The superimposed signal chart measured without people in the room was recorded at the weekend when the building was empty. In the absence of people, the channel still exhibits small variations. The room overlooks a busy road and detailed evaluation of the data showed that reflections from passing vehicles introduce small variations in the signal levels.

There is a strong correlation between the received signal power and the number of people in the room. The average signal power varies about the reference level (when there is nobody in the room). Intuitively it is expected that the presence of people will result in the attenuation of the signal due to shadowing of the LOS path. However periods of sustained enhancements, for example, between 11:30 and 13:30 in Figure 5(a), were often observed at all frequencies. Analysis of the video images and impulse responses showed that these enhancements can be attributed to the scattering of signal components towards the receiver by people standing in the room. Deeper fades, up to $30 \mathrm{~dB}$, were measured than enhancements. Figure 6 shows an example of the distribution of the small-scale signal variation in the presence and absence of people in the room. Significant discrepancies between the Rice K-factors obtained in the presence and absence of people were observed. The values of the K-factor for different antenna types and frequencies are summarised in Table 4.

\section{Channel Impulse Response}

All impulse responses measured in $4 \mathrm{~s}$ were superimposed to obtain the delay power profile (DPP). The statistics of 


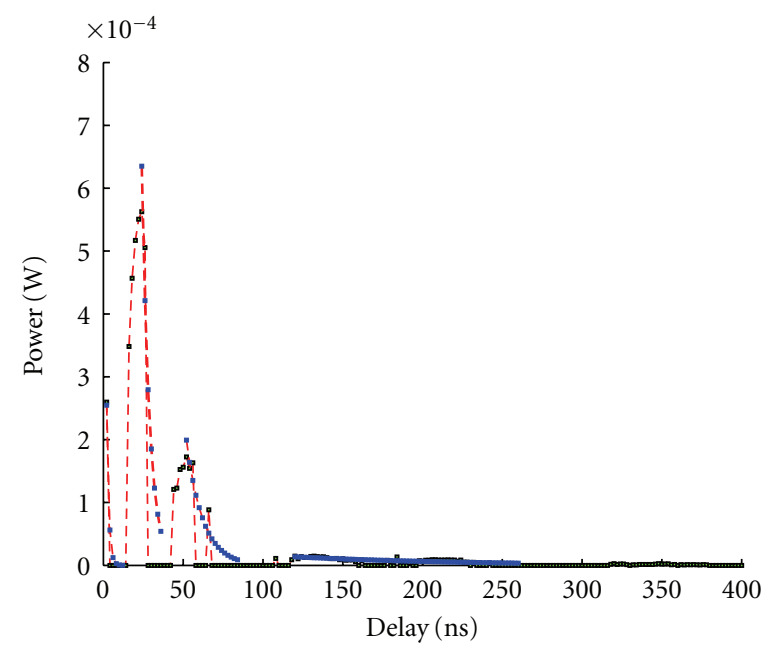

(a)

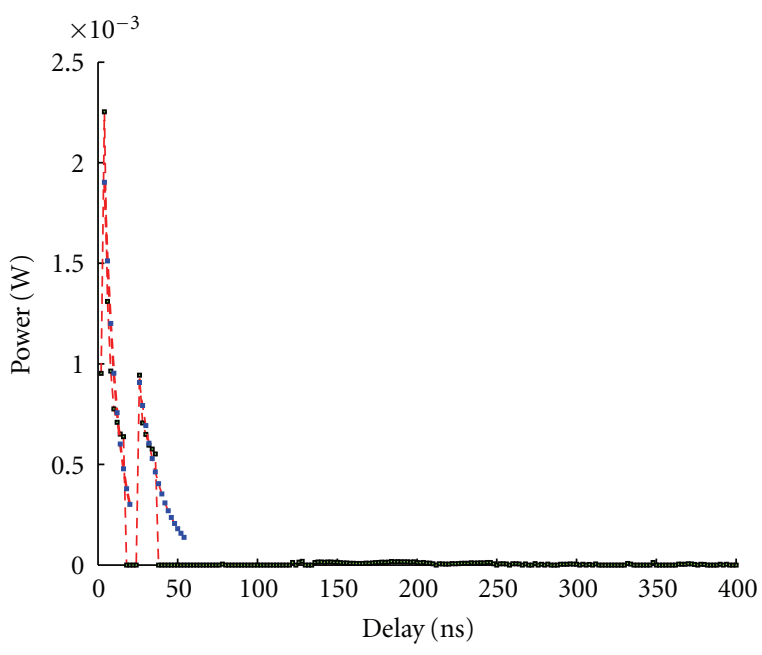

(b)

FIGURE 8: (a) Example where secondary cluster amplitude is larger than the LOS cluster ( 0 ns cluster) and (b) where the DPP follows the SV model (the same room and frequency but different position).

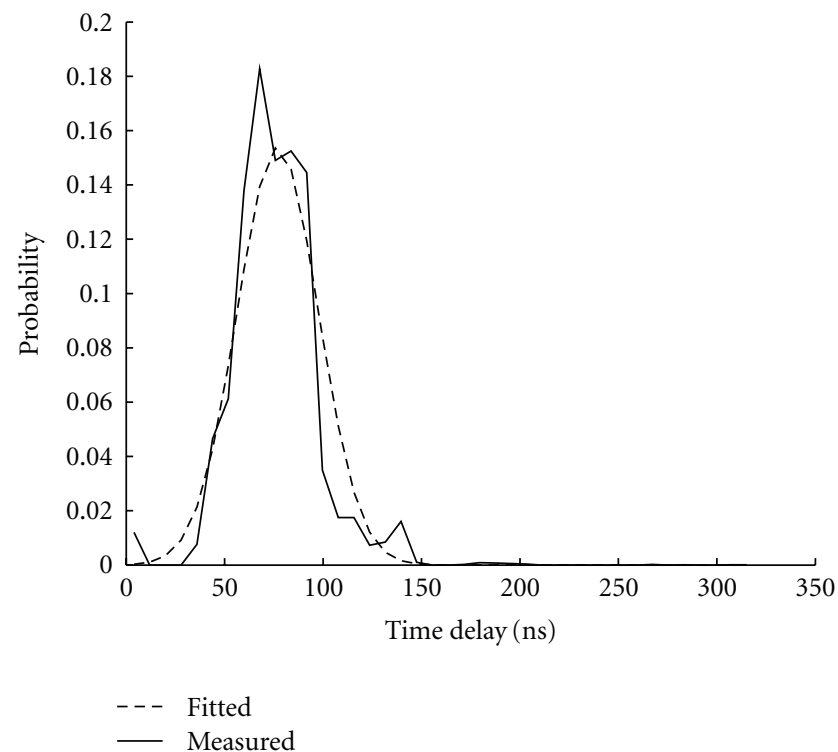

(a)

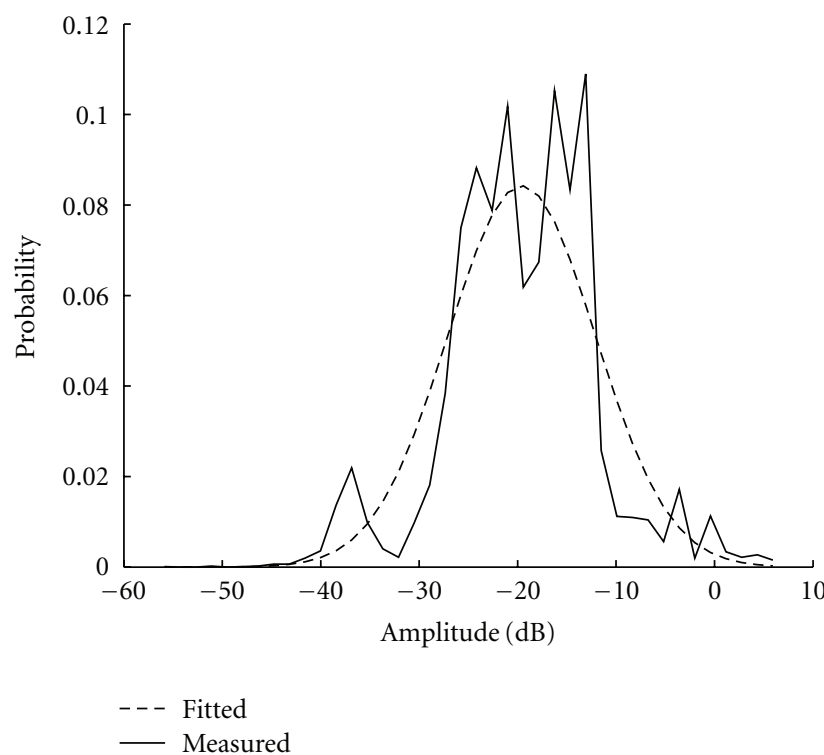

(b)

FIGURe 9: Probability distribution of (a) time of arrival $(\tau)$ and (b) amplitude $(\alpha)$ of multipath components.

the root mean square (RMS) delay spread were computed from the DPP. A summary of the RMS delay spreads from the different locations is given in Table 5. RMS delay spread is shown to decrease with room size (volume). These results confirm the phenomenological explanation of signal propagation within indoor channels. In general, lower values of delay spread are obtained with directional antennas, except for measurements at $1.3 \mathrm{GHz}$. This anomaly can be explained by the fact those rooms have big windows, and signal components reflected from outside the building were received by the directional antenna. It is reported in [16] that the number of significant multipath components for
$31.25 \mathrm{MHz}$ bandwidth is approximately 6 or less. As the rooms become smaller, the ability to resolve the multipath components diminishes.

5.1. Impulse Response Modelling. The results have shown that multipath components tend to form clusters, which has been modelled using the Saleh-Valenzuela (SV) model [6]. To identify the clusters, the impulse responses measured at each position were superimposed to obtain a single delay power profile. The clustering effect can be associated with the building superstructure that includes the walls, the 


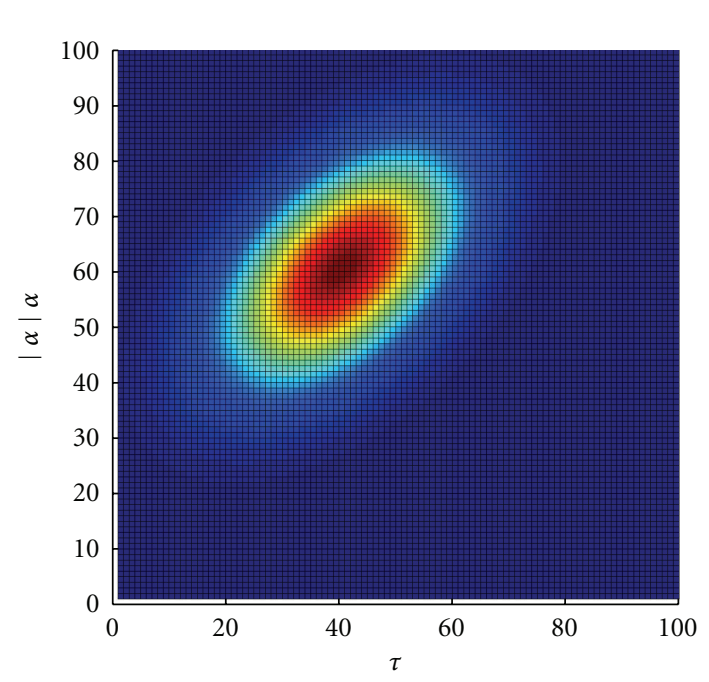

(a)

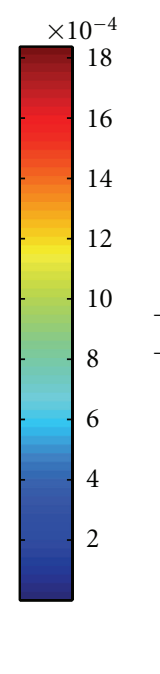

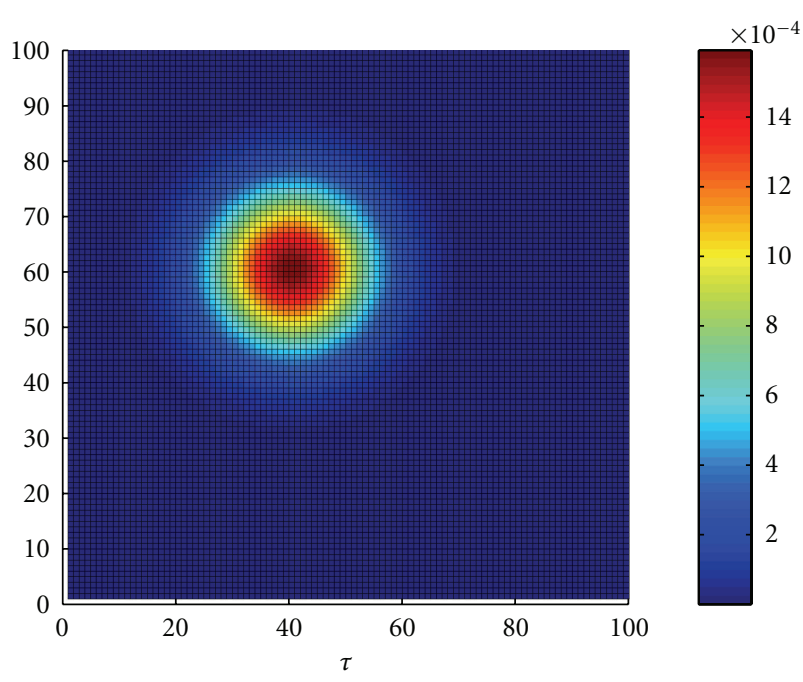

(b)

FIGURE 10: Dependency of joint amplitude and delay distributions on the shape factor: (a) $r=0.5$ (b) $r=0$.

TABLE 4: Rician k-factor for measurements conducted in the presence and absence of people.

\begin{tabular}{|c|c|c|c|}
\hline Frequency GHz & Antenna & Presence of People & Average k-factor (dB) \\
\hline \multirow{4}{*}{1.3} & \multirow{2}{*}{ Omni-directional } & With & 6.3 \\
\hline & & Without & 5.1 \\
\hline & \multirow{2}{*}{ Directional } & With & 2.7 \\
\hline & & Without & 49 \\
\hline \multirow{4}{*}{2} & \multirow{2}{*}{ Omni-directional } & With & 14.7 \\
\hline & & Without & 49 \\
\hline & \multirow{2}{*}{ Directional } & With & 5 \\
\hline & & Without & 19.9 \\
\hline \multirow{4}{*}{11.6} & \multirow{2}{*}{ Omni-directional } & With & 4.1 \\
\hline & & Without & 4.7 \\
\hline & \multirow{2}{*}{ Directional } & With & 2.1 \\
\hline & & Without & 4.7 \\
\hline
\end{tabular}

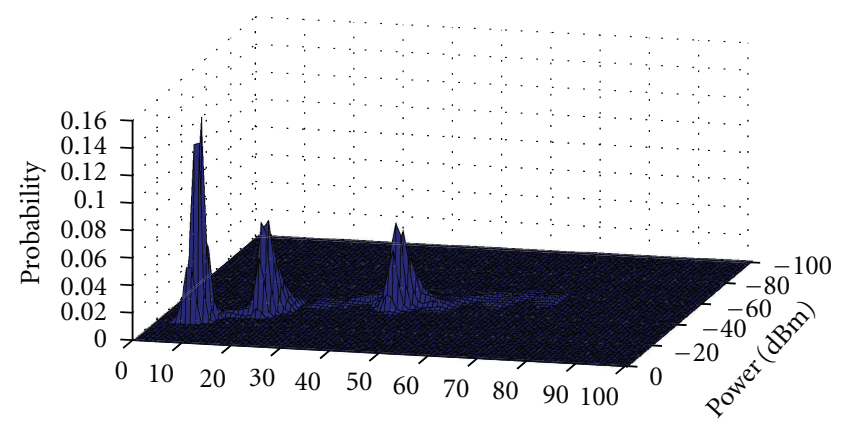

Figure 11: Example of the joint probability distribution of amplitude and time of arrival (measured).

furniture, and the surrounding environment. The SV model is represented by

$$
\overline{\beta_{k l}^{2}}=\overline{\beta^{2}(0,0)} e^{-\tau_{l} / \Gamma} e^{-\tau_{k l} / \gamma}
$$

where $\beta_{k l}$ is the power of the $l$ th multipath component (ray) in the $k$ th cluster, $\overline{\beta^{2}(0,0)}=\overline{\beta_{00}^{2}}$ is the average power of the first ray inside the first cluster and, $\Gamma$ and $\gamma$ are the power decay constants of the clusters and rays, respectively. The decay of the clusters and rays are modelled using exponential functions

$$
\begin{gathered}
p\left(\tau_{l} \mid \tau_{l-1}\right)=\Lambda_{\text {mean }} e^{-\Lambda_{\text {mean }}\left(\tau_{1}-\tau_{l-1}\right)}, \\
p\left(\tau_{k l} \mid \tau_{k-1}\right)=\lambda_{\text {mean }} e^{-\lambda_{\text {mean }}\left(\tau_{k l}-\tau_{k-1}\right)},
\end{gathered}
$$

where $\lambda_{\text {mean }}$ and $\Lambda_{\text {mean }}$ are the arrival rates of the rays and clusters, respectively.

The building surfaces (walls and floors) are assumed to act as strong reflecting and scattering surfaces that give rise to multipath components. In the analysis carried out, and from previous studies using SVDP algorithm, each ray is assumed to be a cluster of irresolvable multipath components. An example of measured DPP is shown in Figure 7.

The average number of clusters obtained under LOS conditions was 3 . This number is smaller than reported in 


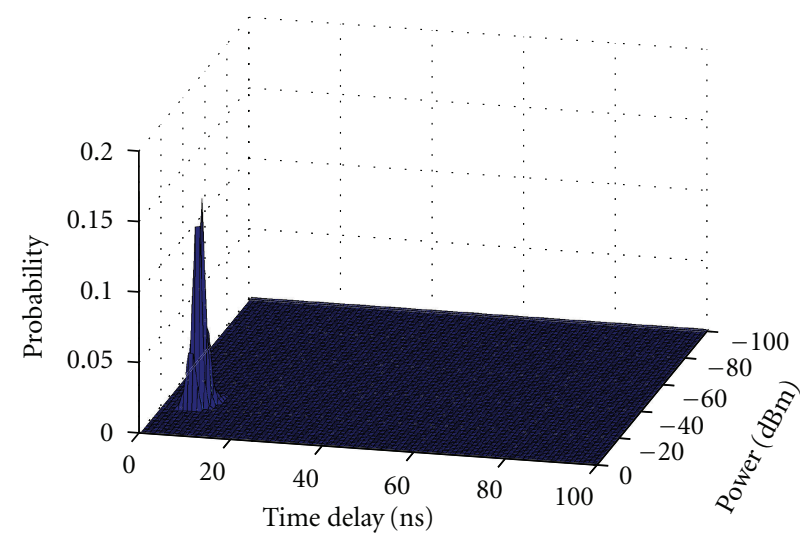

(a)

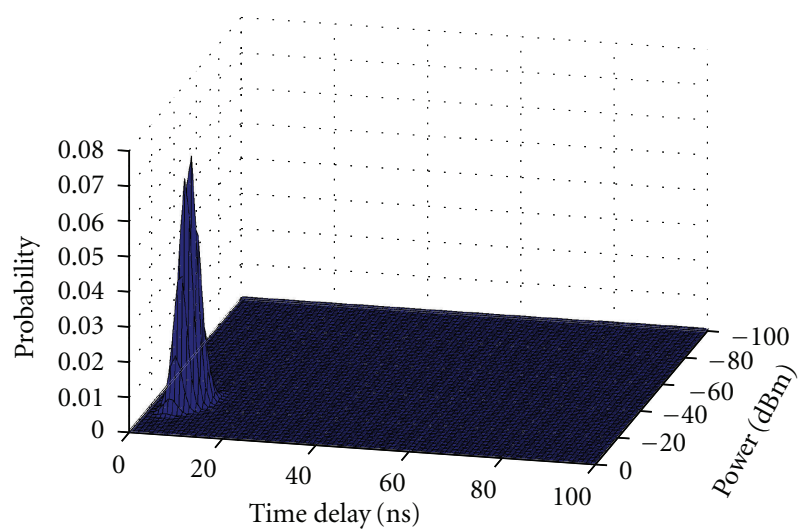

(b)

Figure 12: Joint distribution of $\tau$ and $\alpha$ (a) measured and (b) estimated by model.

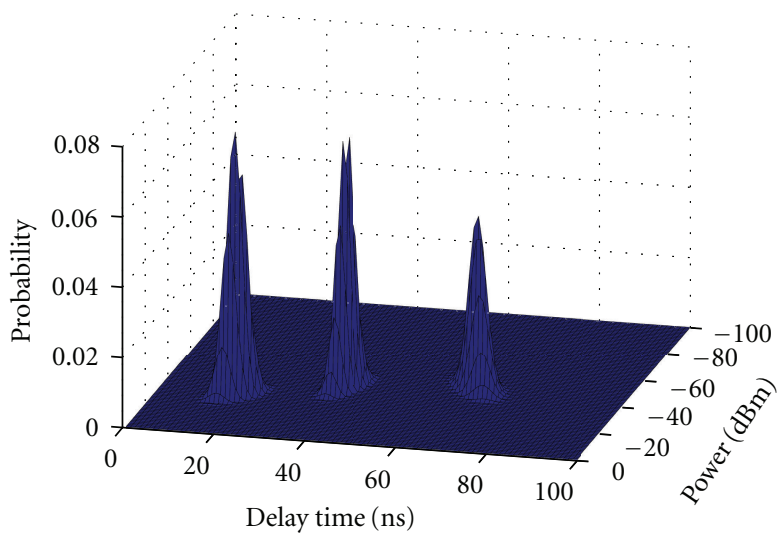

(a)

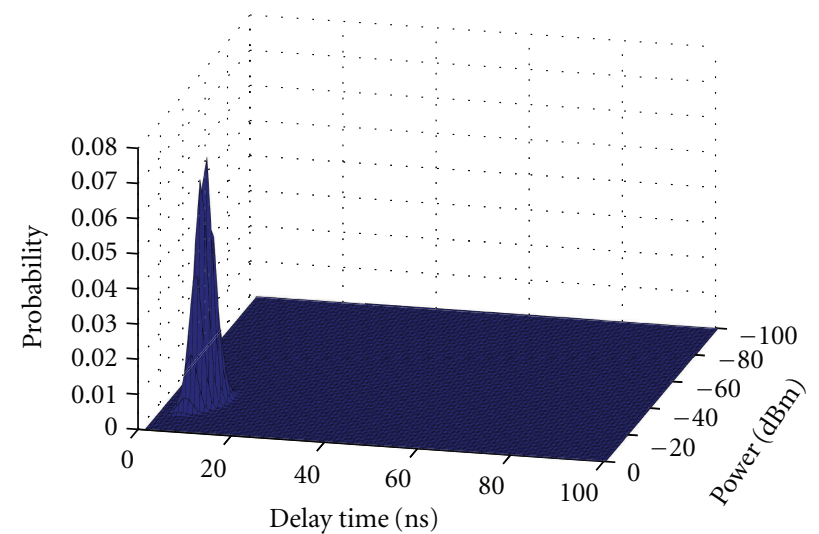

(b)

FIGURE 13: $2 \mathrm{GHz}$ omni-directional antenna measurements with people (a) fitted, (b) measured responses.

[2] where a combination of a $120 \mathrm{MHz}$ bandwidth sounder and a superresolution algorithm was used. The distribution of the number of clusters identified in this study is consistent with results reported in $[6,17]$.

Whilst there is a general agreement that the SV model can be used in most cases, in some instances the fundamental assumptions of the model was not supported by experimental data; for example, when the amplitude of the first cluster is smaller than that of the second cluster, as shown in Figure 8(a). Thus the global decay profile of the DPP with time does not fit the exponential distribution as specified in the SV model. This has been found to depend on the receiver position and the environment. A stronger secondary cluster can be attributed to signals reflected from multiple walls or surfaces that combine to give a more powerful cluster than the LOS component. An example of measurement results that conforms to the SV assumption is given in Figure 8(b). The dotted lines (blue) represent the fitting of the SV model to the clusters. Overall, the results show that the number of clusters decreases with room size.
5.2. Joint Modelling of Amplitude and Delay of Multipath Clusters. One of the main difficulties in the modelling of channel impulse response (CIR) is to estimate the amplitude of the signal component. If the CIR can be considered to be described by (8), where $k$ is the number of clusters and $m_{k}$ is the number of impulses within the $k$ th cluster, $\alpha_{j_{k}}$ and $\tau_{j_{k}}$ are the amplitude and time delay of the $j$ th impulse in the $k$ th cluster, and $n\left(t_{k+j_{k}}\right)$ is zero-mean Gaussian noise at time $k+j_{k}$; then from this model a matrix of the parameters, $\alpha$ and $\tau$ can be obtained that fully describes the impulse response of the channel

$$
\rho(t)=\sum_{k=0}^{k_{\max }} \sum_{j_{k}}^{m_{k}} \alpha_{j_{k}} s\left(t_{k}-\tau_{j_{k}}\right) e^{j \theta_{j_{k}}}+n\left(t_{k+j_{k}}\right) .
$$

The amplitude $(\alpha)$ and delay $(\tau)$ can be described by probability distributions that can be estimated from measured values. Using radio wave propagation principles, the time delay of multipath components can easily be determined for a given path length. However, the amplitude of each 
TABLE 5: Mean RMS delay spread (ns) in different indoor channels.

\begin{tabular}{|c|c|c|c|c|c|c|c|}
\hline \multirow{2}{*}{ Room } & \multirow{2}{*}{ Volume $\left(\mathrm{m}^{3}\right)$} & \multicolumn{2}{|c|}{$1.3 \mathrm{GHz}$} & \multicolumn{2}{|c|}{$2 \mathrm{GHz}$} & \multicolumn{2}{|c|}{$11.6 \mathrm{GHz}$} \\
\hline & & Omni-Directional & Directional & Omni-Directional & Directional & Omni-Directional & Directional \\
\hline Canteen & 1054.5 & 33.3 & 34.9 & 26.2 & 25.3 & 39.2 & 40 \\
\hline A2.2 & 347.8 & 30 & 28 & 21.2 & 18 & 36.6 & 34.1 \\
\hline A2.9 & 250.9 & 23.7 & 21.8 & 21.4 & 20.8 & 21.8 & 16.3 \\
\hline Staff room & 238.8 & 24 & 28 & 15 & 9.6 & 26.3 & 20.3 \\
\hline A1.4 & 217.6 & 15.9 & 21.8 & 10 & 9 & 27.2 & 21.6 \\
\hline A2.4 & 199.4 & 18.9 & 22 & 12.1 & 12.5 & 29.5 & 22.8 \\
\hline A1.9 & 79.1 & 18.1 & 23.3 & 12.1 & 13.3 & 22.2 & 22.3 \\
\hline Corridor 1 & $>1000$ & 30.7 & 28.8 & 18.8 & 16.7 & 45.9 & 47.5 \\
\hline Corridor 2 & $>1000$ & 37 & 34 & 19.3 & 19.1 & 46.5 & 43 \\
\hline
\end{tabular}

component cannot be easily estimated because it depends on the total propagation path length, mode of signal propagation, and the physical and electrical properties of the objects within the channel. However, if the distribution of $\alpha$ and $\tau$ can be jointly modelled, it will enable the amplitude of a ray to be generated based on its time of arrival for a given environment.

Figure 9 shows an example of a joint distribution of experimentally obtained $\tau$ and $\alpha$ in an indoor environment. It can be seen that the two parameters can be modelled using normal probability density functions. If both amplitude and time of arrival are normally distributed, then the joint distribution of $\alpha$ and $\tau$ can be modelled using

$$
\begin{aligned}
& f(\alpha, \tau) \\
& =\frac{1}{2 \pi \sigma_{\alpha} \sigma_{\tau} \sqrt{1-r^{2}}} e \\
& \quad \times\left\{\frac{1}{2\left(1-r^{2}\right)}\left(\frac{\left(\tau-\eta_{\tau}\right)^{2}}{\sigma_{\tau}^{2}}-2 r \frac{\left(\tau-\eta_{\tau}\right)\left(\alpha-\eta_{\alpha}\right)}{\sigma_{\alpha} \sigma_{\tau}}+\frac{\left(\alpha-\eta_{\alpha}\right)^{2}}{\sigma_{\alpha}^{2}}\right)\right\},
\end{aligned}
$$

where $\sigma_{\alpha}$ and $\sigma_{\tau}$ are the standard deviations of $\alpha$ and $\tau$, and $\eta_{\alpha}$ and $\eta_{\tau}$ are the mean values of $\alpha$ and $\tau$, respectively. The parameter $r$ is the shape factor, which gives the angular spread of the distribution of $\alpha$ and $\tau$. The dependency of the joint distribution on $r$ is shown in Figure 10. The model values of the shape factor $(r)$ were found to be between -0.5 and 0.5 for all measurements. Figure 11 shows an example of the joint distribution of the amplitudes and times of arrival of three distinct clusters.

To evaluate the joint amplitude and delay distribution, a data processing procedure was applied to the measured data to identify all the clusters in the DPP. The identified clusters were then used to evaluate the model. An example of an extracted cluster and its estimate based on the model is shown in Figure 12. The model has been applied to measured data in both static and dynamic channels.

The discrepancies in the value of the probabilities (heights of cluster) between the measured and fitted clusters in Figure 12 can be attributed to the fact that the model assumes a continuous distribution of power. However, some bins within the extracted clusters have lower power because of the high delay resolution used.

For all measurements, the standard deviation of the amplitudes and time of arrival increased by a factor of 2 at all frequencies when there were people within the channel. Figure 13 shows an example of measured and modelled DPP.

\section{Conclusions}

Extensive measurement results at 1.3, 2, and 11.6 GHz have been reported. The Singular Value Decomposition Prony algorithm that has been used to calculate the channel impulse response from measured transfer function achieves time delay resolution better than $5 \mathrm{~ns}$ for a $31.25 \mathrm{MHz}$ measurement bandwidth. This high resolution has enabled a full assessment of multipath cluster structure in indoor channels. The use of a sounder with a larger bandwidth would provide higher impulse response resolution that could be used to identify subclusters. However, the resolution achieved in this study has been considered sufficient to identify the influence of structures with dimensions of approximately $1.5 \mathrm{~m}$. It has been shown that the structure of the clusters depends on the objects and surfaces in the environment.

Detailed analysis of indoor measurements in the presence of people has shown that temporal dynamic signal variations of more than $20 \mathrm{~dB}$ are often observed. The signal variations manifest as enhancement or fading, compared to levels obtained in the absence of people. This is similar to the results found in previous research [4]. Severe fading, up to $40 \mathrm{~dB}$, has been measured when there were people in close proximity to the transmitting antenna in the LOS path. Further analysis confirmed that the impact of people on the channel characteristics depends more on their positions rather than on the total number within the channel. However, it has been found that the random scattering of signal by people in the channel provides opportunities to achieve high reception diversity gain.

A joint probability density function that can be used to jointly model the amplitude and the time of arrival of multipath components has been proposed. The proposed model incorporates a scaling factor that determines the shape of the multipath cluster. The proposed model can be used 
to simulate indoor channel response to evaluate current and future wireless communication systems.

\section{Acknowledgment}

This work was funded by UK EPSRC Grant No. GR/ N03174/ 01 .

\section{References}

[1] A. Doufexi, E. Tameh, A. Nix, S. Armour, and A. Molina, "Hotspot wireless LANs to enhance the performance of $3 \mathrm{G}$ and beyond cellular networks," IEEE Communications Magazine, vol. 41, no. 7, pp. 58-65, 2003.

[2] C.-C. Chong, C.-M. Tan, D. I. Laurenson, S. McLaughlin, M. A. Beach, and A. R. Nix, "A new statistical wideband spatiotemporal channel model for 5-GHz band WLAN systems," IEEE Journal on Selected Areas in Communications, vol. 21, no. 2, pp. 139-150, 2003.

[3] Y. Chen and V. K. Dubey, "Dynamic simulation model of indoor wideband directional channels," IEEE Transactions on Vehicular Technology, vol. 55, no. 2, pp. 417-430, 2006.

[4] J. D. Gupta, H. Suzuki, and K. Ziri-Castro, "Effect of pedestrian movement on MIMO-OFDM channel capacity in an indoor environment," IEEE Antennas and Wireless Propagation Letters, vol. 8, pp. 682-685, 2009.

[5] G. Calcev, D. Chizhik, B. Göransson et al., "A wideband spatial channel model for system-wide simulations," IEEE Transactions on Vehicular Technology, vol. 56, no. 2, pp. 389403, 2007.

[6] A. A. M. Saleh and R. A. Valenzuela, "Statistical model for indoor multipath propagation," IEEE Journal on Selected Areas in Communications, vol. 5, no. 2, pp. 128-137, 1987.

[7] J. Austin, W. P. A. Ditmar, W. K. Lam, and E. Vilar, "A spread spectrum communications channel sounder," IEEE Transactions on Communications, vol. 45, no. 7, pp. 840-847, 1997.

[8] A. Hewitt, W. H. Lau, J. Austin, and E. Vilar, "Autoregressive approach to the identification of multipath ray parameters from field measurements," IEEE Transactions on Communications, vol. 37, no. 11, pp. 1136-1143, 1989.

[9] C. Williams, M. Beach, D. Neirynck et al., "Personal area technologies for internetworked services," IEEE Communications Magazine, vol. 42, no. 12, pp. S15-S26, 2004.

[10] J. Medbo and P. Schramm, "Channel models for HIPERLANS/2 in different indoor scenarios," 3ERI085B, HIPERLANS/2 ETSI/BRAN contribution, March, 1998.

[11] ITU-R Recommendation P.1238-2, 2001.

[12] F. Aguado Agelet, A. Martínez, J. M. Hernando, F. Isasi, and D. Mosteiro, "Bundle method for optimal transmitter location in indoor wireless system," Electronics Letters, vol. 36, no. 6, pp. 573-574, 2000.

[13] S. Y. Seidel and T. S. Rappaport, "914 MHz path loss prediction models for indoor wireless communications in multifloored buildings," IEEE Transactions on Antennas and Propagation, vol. 40, no. 2, pp. 207-217, 1992.

[14] D. Molkdar, "Review on radio propagation into and within buildings," IEE Proceedings H, vol. 138, no. 1, pp. 61-73, 1991.

[15] A. F. Abou-Raddy and S. M. Elnoubi, "Statistical modeling of the indoor radio channel at $10 \mathrm{GHz}$ through propagation measurements-part 1: narrow-band measurements and modelling," Wireless Networks, vol. 49, no. 5, pp. 1491-1507, 2000.
[16] D. Ndzi, J. Austin, and E. Vilar, "Hyper-resolution indoor channel impulse responses: multipath components and $\mathrm{k}$ factors," Electronics Letters, vol. 35, no. 9, pp. 698-699, 1999.

[17] Q. H. Spencer, B. D. Jeffs, M. A. Jensen, and A. L. Swindlehurst, "Modeling the statistical time and angle of arrival characteristics of an indoor multipath channel," IEEE Journal on Selected Areas in Communications, vol. 18, no. 3, pp. 347-360, 2000. 

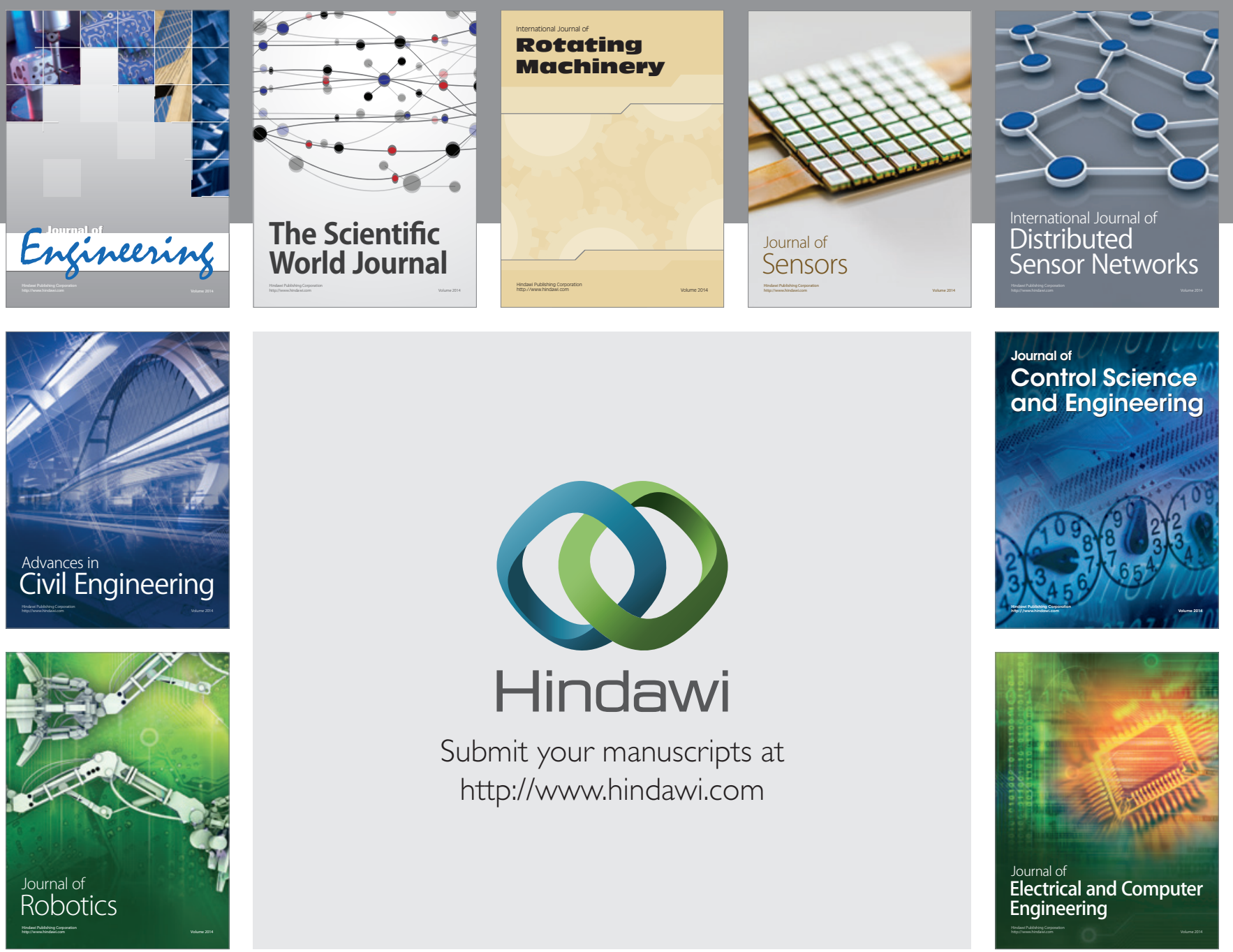

Submit your manuscripts at

http://www.hindawi.com
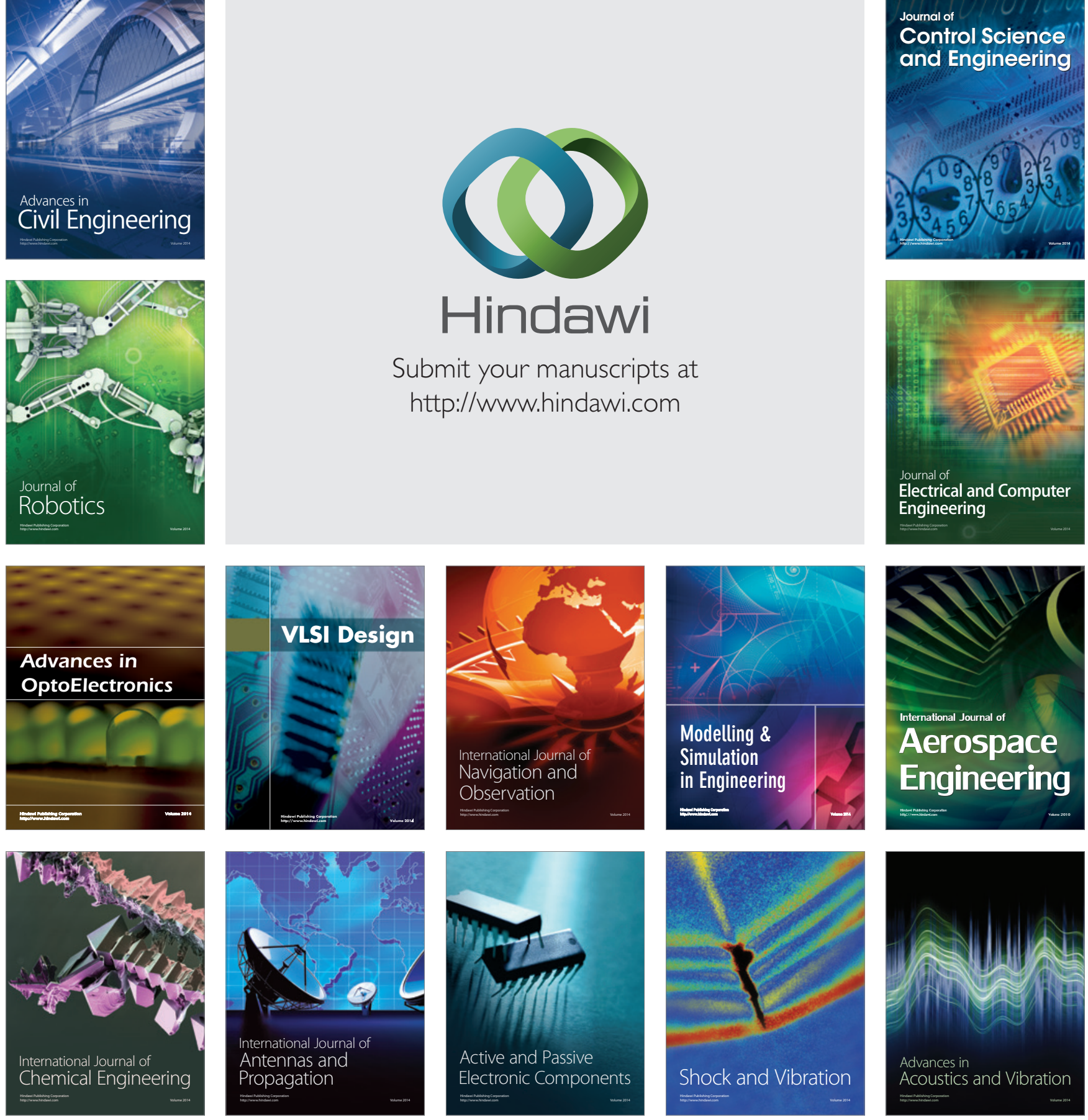